\title{
GOÄ-Ausschluss? Auslagen trotzdem berechnen!
}

— $\$ 10$ der GOÄ sieht vor, dass neben den für die einzelnen ärztlichen Leistungen vorgesehenen Gebühren auch Auslagen berechnet werden können. Das sind Kosten für Arzneimittel, Verbandmittel und sonstiges Materialien, die der Patient zur weiteren Verwendung behält oder die mit einer einmaligen Anwendung verbraucht sind, soweit in Absatz 2 nichts anderes bestimmt ist. Als Auslagen gelten auch Versand- und Portokosten, soweit deren Berechnung nach Absatz 3 nicht ausgeschlossen ist. Ferner berechnet werden können Kosten, die im Zusammenhang mit Leistungen nach Abschnitt $\mathrm{O}$ bei der Anwendung radioaktiver Stoffe durch deren Verbrauch entstehen. Daneben gibt es natürlich noch Kosten, die explizit in der GOÄ als gesondert berechnungsfähig ausgewiesen sind.

Die Berechnung von Pauschalen ist nach $₫ 10$ zwar nicht zulässig, aber fast alle Versicherungen akzeptieren der Einfachheit halber den Nebenkostentarif der Unfallversicherung (UV-GOÄ). Die Kosten können sogar dann berech- net werden, wenn die zugrunde liegende Abrechnungsposition wegen Ausschlüssen nicht zum Ansatz kommt.

\section{MMW-KOMMENTAR}

Dieser Fall dürfte in der hausärztlichen Praxis keineswegs selten sein. In der GOÄ dürfen z. B. die Nrn. 1 oder 5 nur einmal im Monat neben einer Leistung nach den Abschnitten $\mathrm{C}-\mathrm{O}$ berechnet werden. Betroffen sind hier häufig kleinchirurgische Leistungen wie Wundversorgungen. Diese sind im Laufe eines solchen Monats oft mehrfach notwendig - und fallen dann neben den Nrn. 1 und 5 weg. Schließlich bringen diese bei Abrechnung mit dem 2,3-fachen Faktor 21,42 Euro, wogegen der Verbandswechsel nach Nr. 200 nur mit 6,03 Euro vergütet wird. Damit keine unnötigen Rückfragen entstehen, empfiehlt es sich hier aber, die Nr. 200 in der Rechnung mit dem Faktor 0 und damit 0 Euro anzusetzen. So hängt die Berechnung der Kosten für den Verband nicht "in der Luft". Nach dem aktuellen Stand der UV-GOÄ können für das Verbandsmaterial 1,28 Euro als Auslagenersatz in Rechnung gestellt werden.

\section{Tab. 2 Beispiele für berechnungs- fähige Kosten bei häufigen Leistungen auf Grundlage des Nebenkostentarifs der UV-GOÄ}

\begin{tabular}{|l|l|l|}
\hline GOÄ & Legende & \multicolumn{1}{|c|}{ Euro } \\
\hline 200 & Verband & 1,28 \\
\hline $203 \mathrm{~A}$ & Kompressionsverband & 3,88 \\
\hline $203 \mathrm{~B}$ & Zinkleimverband & 15,77 \\
\hline 210 & Kleiner Schienenverband & 5,43 \\
\hline 490 & Infiltrationsanästhesie klein & 1,48 \\
\hline 491 & Infiltrationsanästhesie groß & 2,59 \\
\hline 493 & $\begin{array}{l}\text { Oberstsche Leitungs- } \\
\text { anästhesie }\end{array}$ & 1,73 \\
\hline 2001 & $\begin{array}{l}\text { Versorgung einer kleinen } \\
\text { Wunde einschließlich Naht }\end{array}$ & 5,41 \\
\hline 2001 & $\begin{array}{l}\text { Versorgung einer kleinen } \\
\text { Wunde durch Gewebekleber }\end{array}$ & 8,50 \\
\hline 2002 & $\begin{array}{l}\text { Versorgung einer kleinen } \\
\text { Wunde einschließlich } \\
\text { Umschneidung und Naht }\end{array}$ & 5,47 \\
\hline 2004 & $\begin{array}{l}\text { Versorgung einer großen } \\
\text { Wunde einschließlich Naht }\end{array}$ & 9,40 \\
\hline & & \\
\hline
\end{tabular}

\section{KBV-Vorstand will Immobilienaffäre abschließen}

In einem Schreiben vom 22. September 2015 wendet sich KBV-Chef Dr. Andreas Gassen an die Mitglieder der Vertreterversammlung (VV). Der Brief bezieht sich auf die KBV-VV am 18. September 2015, bei der auch die Causa ApoBank-Vermietung wieder thematisiert wurde. Gemeint ist ein komplizierter Sachverhalt, der die KBV seit Jahren belastet: Das Gebäude der Körperschaft in Berlin wurde von einer Tochtergesell- schaft der Deutschen Apotheker- und Ärztebank gebaut und zunächst von der KBV angemietet, bevor diese die Gesellschaft mehrheitlich übernahm. Dabei lief offenbar Einiges schief; unterm Strich könnte den Vertragsärzten ein Millionenschaden entstanden sein.

Gassen stellt nun fest, dass die Vergangenheit aufgearbeitet werden müsse und erste Schritte bereits eingeleitet seien. $\mathrm{Ob}$ eine Strafanzeige notwendig sei, müsse noch geklärt werden. Er stellt aber fest, dass die ApoBank-Vermietung bereits intensiv und in enger Abstimmung mit dem Bundesgesundheitsministerium von einer Wirtschaftsprüfungsgesellschaft unter die Lupe genommen worden sei. Gassen schließt das Schreiben zuversichtlich, dass man nach der Aufarbeitung der Vergangenheit die Zukunft gemeinsam positiv gestalten könne. Er bittet diesbezüglich um Ideen und Wünsche. 